\title{
Recuperación mejorada en cirugía bariátrica, ¿Una verdadera revolución?
}

\section{Enhanced recovery after surgery, a true revolution?}

\author{
Florencia Estefanía Bolla1,* \\ 1 Hospital Italiano de Buenos Aires. Buenos Aires, Argentina.
}

Fecha de recepción: 27 de abril de 2021 / Fecha de aceptación: 17 de mayo de 2021

\begin{abstract}
The ERAS (Enhanced Recovery After Surgery) program proposes a cultural challenge that implies a change from traditional patient care to an evidence-based care, with the main goal of improving perioperative outcomes. It consists of a multidisciplinary approach with patient centered care, which encompasses preoperative, intraoperative and postoperative care. In bariatric surgery, it has demonstrated a reduction in the incidence of nausea and vomiting, better oral tolerance, a minimized stress response and less insulin resistance. Moreover, it has showed an opioid spearing effect, together with a reduction in hospital length of stay and re-admissions.
\end{abstract}

Key words: ERAS, bariatric surgery.

\section{RESUMEN}

El programa ERAS (Enhanced Recovery After surgery) es una propuesta de cambio cultural que integra una migración del cuidado tradicional del paciente a un cuidado basado en la evidencia, con el objetivo de optimizar los outcomes perioperatorios. Propone un abordaje multidisciplinario centrada en el paciente, que abarca los aspectos del preoperatorio, intraoperatorio y posoperatorio; donde el paciente cobra un rol completamente activo en su recuperación. En cirugía bariátrica, ha demostrado disminuir la incidencia de náuseas y vómitos, mayor tolerancia a la vía oral y una minimización de la respuesta al stress como de la resistencia a la insulina. A su vez, ha evidenciado un efecto ahorrador de opioides, una disminución en la estadía hospitalaria y en las readmisiones.

Palabras clave: ERAS, cirugía bariátrica.

\section{Introducción}

E n la actualidad, la implementación del programa ERAS (Enhanced Recovery After Surgery) se ha convertido en la columna vertebral de muchos hospitales de referencia a lo largo del mundo entero. Los invito a reflexionar sobre el concepto más profundo que trae aparejado la implementación de dichos programas. El programa ERAS es una propuesta de cambio cultural con una migración del cuidado tradicional del paciente a un cuidado basado en la evidencia, con el objetivo de optimizar los outcomes perioperatorios. La palabra programa refleja un concepto mucho más amplio y rico que la palabra protocolo, ya que efectivamente ERAS es mucho más que cumplir checklists.

Históricamente, la organización hospitalaria convencional ha sido diseñada como una serie de silos, es decir, unidades o departamentos independientes y aislados entre sí, donde reinaba la una ausencia de comunicación entre el departamento de Clínica Médica, Cirugía, Anestesia y Cuidados Intensivos. Ciertamente, el programa ERAS propone la implementación de una revolución en la comunicación dentro de la comunidad médica, que deviene en una nueva forma de cuidado de nuestros pacientes. En el 2007, la Joint Comission International, una organización dedicada a la acreditación de centros sanitarios en función de determinados estándares de cuidado del paciente, reportó que la comunicación ineficiente era la causa del $65 \%$ 
de los errores clínicos. Y en el 2009 la agregó como una de las 5 metas para garantizar la seguridad del paciente.

Por su parte, ERAS propone una integración multidisciplinaria centrada en el paciente, que abarca los aspectos del preoperatorio, intraoperatorio y posoperatorio; donde el paciente cobra un rol completamente activo en su recuperación, con un gesto de rechazo a la medicina paternalista.

¿Por qué deberíamos implementar este programa en pacientes de cirugía bariátrica? Porque ha demostrado disminución de la incidencia de náuseas y vómitos, mayor tolerancia a la vía oral, una minimización de la respuesta al stress como de la resistencia a la insulina. A su vez, se ha demostrado un efecto ahorrador de opioides, disminución en la estadía hospitalaria y en las readmisiones. Todo lo antedicho es fundamental en una población que se caracteriza por presentar resistencia a la insulina, que tiene aumento de incidencia de episodios de deshidratación posoperatorios y presenta suturas en estómago propio del procedimiento quirúrgico.

\section{Implementación}

El proceso del cuidado del paciente se basa en tres pilares fundamentales:

- Comunicación efectiva: a través de reuniones de equipo multidisciplinarias, programadas, con un gran compromiso en su asistencia y participación.

- Consenso de cuidados: cumplimiento de las guías de cuidado aceptadas y validadas por toda la comunidad médica participante.

- Control de resultados: basado en un sistema de auditorías continua.

En referencia a las guías de cuidado propiamente dichas, la ERAS Society publicó en el año 2016 una guía en relación a cirugía bariátrica. A continuación, se destacan las medidas con distinto nivel de evidencia, pero con un nivel de recomendación fuerte por parte de los expertos.

La ERAS Society recomienda fuertemente:

Con respecto al momento preoperatorio:

- Counselling preoperatorio.

- Cesación de alcohol y tabaco 4 semanas previas a la cirugía.

- Dieta baja en calorías al menos durante 2 semanas.

- Administración de dexametasona endovenosa 90 minutos previo a la inducción anestésica.

- Ayuno de líquidos de $2 \mathrm{~h}$ y de sólidos de 6 h.

- Tromboprofilaxis mecánica y endovenosa.

Con respecto al momento intraoperatorio:

- Abordaje multimodal de náuseas y vómitos.

- Evitar ansiolíticos y opioides de larga duración.

- Analgesia multimodal endovenosa con infiltración de los puertos laparoscópicos con anestésicos locales.

- Ventilación mecánica protectiva.

- Monitoreo de la hipnosis medida por Bispectral index (BIS).

- Reversión total de la relajación neuromuscular, con monitoreo cualitativo de la misma.

Con respecto al momento postoperatorio:

- Suspensión de fluidoterapia endovenosa lo antes posible para rotar a vía oral. Ingesta de líquido la noche de la cirugía, y sólidos al día siguiente.

- No dejar colocada sonda nasogástrica.

Estas recomendaciones son simplemente una parte pequeña del cambio que propone ERAS. Sin bien existen guías establecidas para el cuidado del paciente, el objetivo de optimizar los outcomes perioperatorios se logra con un cuidado centrado en el paciente, con auditorías regulares, en un contexto de comunicación institucional multidisciplinaria, donde la verdadera revolución es efectivamente la comunicación.

Toda ruptura de hábito, por mínima que parezca, produce un cambio si nuestro accionar es consistente y duradero. Hacer mejores preguntas nos lleva a obtener mejores respuestas, por lo cual los invito a preguntarse qué podemos hacer para modificar la experiencia hospitalaria de nuestros pacientes.

\section{Referencias}

1. Thorell A, MacCormick AD, Awad S, Reynolds N, Roulin D, Demartines N, et al. Guidelines for Perioperative Care in Bariatric Surgery: Enhanced Recovery After Surgery (ERAS) Society Recommendations. World J Surg. 2016 Sep;40(9):2065-83. https://doi. org/10.1007/s00268-016-3492-3 PMID:26943657

2. King $A B$, Spann MD, Jablonski P, Wanderer JP, Sandberg WS, McEvoy MD. An enhanced recovery program for bariatric surgical patients significantly reduces perioperative opioid consumption and postoperative nausea. Surg Obes Relat Dis. 2018 Jun;14(6):849-56. https://doi.org/10.1016/j.soard.2018.02.010 PMID:29555468

3. Ahmed OS, Rogers AC, Bolger JC, Mastrosimone A, Robb WB. Meta-Analysis of Enhanced Recovery Protocols in Bariatric Surgery. J Gastrointest Surg. 2018 Jun;22(6):964-72. https://doi. org/10.1007/s11605-018-3709-x PMID:29488124

4. Dang JT, Szeto VG, Elnahas A, Ellsmere J, Okrainec A, Neville A, et al. Canadian consensus statement: enhanced recovery after surgery in bariatric surgery. Surg Endosc. 2020 Mar;34(3):1366-75. https://doi.org/10.1007/s00464-019-06911-x PMID:31209605

5. Nightingale CE, Margarson MP, Shearer E, Redman JW, Lucas DN, Cousins JM, et al.; Members of the Working Party; Association of Anaesthetists of Great Britain; Ireland Society for Obesity and Bariatric Anaesthesia. Peri-operative management of the obese surgical patient 2015: Association of Anaesthetists of Great Britain and Ireland Society for Obesity and Bariatric Anaesthesia. Anaesthesia. 2015 Jul;70(7):859-76. https://doi. org/10.1111/anae.13101 PMID:25950621

6. American Society of Anesthesiologists Task Force on Perioperative Management of patients with obstructive sleep apnea. Practice guidelines for the perioperative management of patients with obstructive sleep apnea: an updated report by the American Society of Anesthesiologists Task Force on Perioperative Management of patients with obstructive sleep apnea. Anesthesiology. 2014 Feb;120(2):268-86. https://doi.org/10.1097/ ALN.0000000000000053 PMID:24346178

7. Inomata T, Mizuno J, Iwagami M, et al. The impact of Joint Commission International accreditation on time periods in the operating room: A retrospective observational study. PLoS One. 2018;13(9):e0204301. Published 2018 Sep 21. https://doi. org/10.1371/journal.pone.0204301. 